\title{
EDITORIAL
}

\section{Annals of Occupational Hygiene Performance, 2015}

Volume 59 of the Annals of Occupational Hygiene, published in nine issues during the year 2015, continued our history of contribution to the science of exposures at work and their impact on the health of workers.

In 2015, we received 275 submissions, roughly the same as the 278 in 2014, including 226 original research articles. Among the 251 papers for which final publication decisions were made, 90 were accepted, giving us a rejection rate of $64.1 \%$. This relatively high rejection rate helps assure the highest quality of papers appear in the journal and is thus good for readers and the journal.

In 2015, we published 85 research papers, 7 short communications, 2 review articles, and an additional 2 commentaries, 4 editorials, and 2 letters to the Editor. A content analysis of our research and review papers show the Annals to be very strong on exposure assessment (42\%, up from 36\% in 2014), measurement (22\%, up from 19\% in 2014), exposure controls (7\%), plus personal protective equipment (15\%), and the basic sciences underlying occupational hygiene (5\%). Aerosols made up $41 \%$ of the agents addressed, with $19 \%$ tackling chemicals, $13 \%$ physical agents, and $6 \%$ bioaerosols. The editorial team coordinated the collection of 506 peer reviews from 295 individuals, highlighting the huge commitment made by all of our contributors.

Of the two commentaries published, one summarized a large body of research on the effectiveness of anti-vibration gloves on the reduction of segmental vibration exposures; the other considered the relationship between work, smoking, and health, presenting important evidence about the health benefits of work, and how the occupational hygienist can help support those positive impacts.
Our time for papers in review has been monitored using a 6-month running average, giving the mean (median) time to a first decision of 29 (28) days, while time to reach a final decision (after revision and rereview) was 76 (37) days (based on July-December).

In addition to the 1867 members of BOHS receiving the hard copy journal, 2880 institutions were able to access the journal either via a direct institutional subscription or through academic consortia agreements. In 2015, 1217 not-for-profit institutions in 35 developing countries obtained free online access. The average number of full-text downloads per month was 25849.

North America and Europe remain the strongest geographic areas for submissions of original articles, reviews, commentaries, and short communications at 39 and $34 \%$, respectively.

The Annals journal impact factor (JIF) for 2014 was 2.10, a slight increase on the 2013 level of 2.07. Our JIF ranking among other journals classified as 'Public, Environmental and Occupational Health' is currently 60 among 162 journals, and 52 out of 87 journals classified as 'Toxicology'.

2015 saw the retirement, mid-year, of former Chief Editor Trevor Ogden, and Assistant Editors David Bartley and Nick Vaughan. We are enormously grateful for the years of dedication all three have provided and will miss the wealth of their collective experience. Dr Renee Anthony from the University of Iowa joined the Board in July 2015 bringing expertise in aerosols and computational fluid dynamics as well as extensive professional experience in occupational hygiene.

The Annals of Occupational Hygiene continues to perform strongly and make an essential contribution to 
the science of primary prevention of occupational disease. However, the world of work is rapidly changing, not only through new technologies and approaches to health and environmental research but also in the ways in which workers and employers organize production and services. With this in mind, the editorial board has begun a review of the Annals' position and direction with a view to maintaining and growing the relevance and impact of the journal. Your comments and suggestions on this or any other issues relating to the Annals are most welcome.

\section{Noah Seixas, Chief Editor \\ DEOHS, University of Washington, Seattle, WA 98105, USA \\ E-mail: aohed@uw.edu}

